\title{
DECOMPOSIÇÃO DE CULTURAS DE COBERTURA NO SISTEMA PLANTIO DIRETO, MANEJADAS MECÂNICA E QUIMICAMENTE
}

\author{
GUSTAVO N. DOS REIS ${ }^{1}$, CARLOS E. A. FURLANI ${ }^{2}$, ROUVERSON P. DA SILVA ${ }^{3}$, \\ JULIANO R. GERLACH ${ }^{4}$, JORGE W. CORTEZ ${ }^{1}$, DANILO C. C. GROTTA ${ }^{1}$
}

\begin{abstract}
RESUMO: O sistema plantio direto preconiza o uso de culturas de cobertura para produção de massa seca sobre o solo. O presente trabalho teve por objetivo estudar a produção e a taxa de decomposição de duas coberturas vegetais - crotalária juncea (Crotalária juncea L.) e mucunacinza (Stilozobium niveum L) - em três sistemas de manejo (rolo-faca, triturador de palhas e herbicida), e a capacidade de campo efetiva dos manejos. O experimento foi realizado na área do Laboratório de Máquinas e Mecanização Agrícola (LAMMA), do Departamento de Engenharia Rural, UNESP, Jaboticabal - SP, com delineamento em blocos ao acaso, em esquema fatorial 3x2, originando seis tratamentos, com quatro repetições. Foram analisadas as produções de massa seca das culturas de cobertura antes e após o manejo, a velocidade de deslocamento e a capacidade de campo efetiva para cada conjunto (trator-equipamento). As análises dos valores obtidos permitiram verificar que o fator manejo não interfere na decomposição da massa seca das coberturas vegetais e que as duas culturas de cobertura apresentaram massas semelhantes aos 30; 70 e 125 dias após a semeadura, diferindo aos 97 dias, época na qual a crotalária apresentou maior quantidade de massa seca. Aos 30; 51 e 71 dias após o manejo, as massas secas das culturas foram semelhantes. O manejo com herbicida apresentou maior capacidade de campo efetiva.
\end{abstract}

PALAVRAS-CHAVE: mucuna-cinza, Crotalária juncea, capacidade de campo efetiva.

\section{ACTING OF COVERING CULTURES IN NO TILLAGE SYSTEM, HANDLING MECHANICS AND CHEMISTRY}

\begin{abstract}
The no tillage using covering cultures about the production dry mass surface in the soil. The present work had for objective to study the production and acting of two vegetable coverings crotalária juncea (Crotalaria juncea L.) and mucuna-cinza (Stilozobium niveum), in three systems of handlings (roll-knife, crusher of straws and herbicide), and and the effective field capacity. The experiment was accomplished in area of Laboratório de Máquinas e Mecanização Agrícola (LAMMA), of the Departamento de Engenharia Rural, UNESP; Jaboticabal - SP, with experimental design consisted of a factorial array $3 \times 2$ with completely randomized blocks, origin six treatments with four repetitions. They were measuring and analyzed parameters of production of dry mass of the coverings vegetable after the sowing and handling, displacement speed and the effective field capacity execute for each group (tractor-equipment). The analysis of the obtained values allowed verifying that the factor handling didn't interfere in the mass it evaporates of covering vegetable. The cultures present similar masses to the 30; 71 and 125 days after the sowing, differing to the 97 days, was the Crotalaria juncea L. present larger mass earnings. In the powders handling the two cultures have masses similar to the 30; 51 and 71 days after the handling, in other words, during whole the experiment. The handling with herbicide present larger effective field capacity.
\end{abstract}

KEYWORDS: mucuna-cinza, crotalária juncea, effective field capacity.

\footnotetext{
${ }^{1}$ Pós-Graduando, UNESP, Jaboticabal - SP, Bolsista Capes, gn_reis@ yahoo.com.br

${ }^{2}$ Prof. Adjunto, Departamento de Engenharia Rural, UNESP, Jaboticabal - SP. Bolsista de Produtividade CNPq.

${ }^{3}$ Prof. Dr., Departamento de Engenharia Rural, UNESP, Jaboticabal - SP. Bolsista de Produtividade CNPq.

${ }^{4}$ Engenheiro Agrônomo.

Recebido pelo Conselho Editorial em: 18-4-2006

Aprovado pelo Conselho Editorial em: 16-10-2006
} 


\section{INTRODUÇÃO}

O Sistema Plantio Direto (SPD) é caracterizado pela permanência de palha, rotação de culturas e restos vegetais na superfície do solo, que posteriormente são revolvidos apenas na fileira de deposição de sementes e fertilizantes, com o controle de plantas daninhas realizado com a aplicação de herbicidas e pelos efeitos alelopáticos de cada planta de cobertura.

A manutenção de resíduos culturais na superfície do solo no SPD promove condições distintas às propriedades do solo em relação à incorporação promovida pelo sistema de preparo convencional. O revolvimento mínimo do solo leva à decomposição mais lenta e gradual da matéria orgânica, apresentando como consequiência alterações físicas, químicas e biológicas no solo, repercutidas na sua fertilidade e na produtividade das culturas, características já observadas por MOODY et al. (1961).

A diversificação de plantas com sistemas radiculares capazes de explorar diferentes profundidades do solo, com potencial diferenciado de reciclagem, proporciona melhor equilíbrio dos nutrientes e incremento na qualidade e na atividade biológica do solo (EMBRAPA, 1998).

Segundo SILVA et al. (1999), devem ser escolhidas, para cobertura, espécies que produzam grande quantidade de matéria seca, resistentes ao ataque de pragas e moléstias, com sementes uniformes de bom poder germinativo e exigência relativamente baixa quanto ao preparo e fertilidade do solo, rápido crescimento, fácil manejo e sistema radicular profundo, dispensando tratos culturais.

Os meios mecânicos de corte das culturas de cobertura podem variar em função do equipamento disponível na propriedade e das adaptações e regulagens efetuadas nesses equipamentos. Para tanto, poderão ser utilizados equipamentos, como alfanje, foice ou gadanho; triturador de palhas; roçadora ou rolo-faca. No caso de plantas perenes, que rebrotam o ano todo, os cortes poderão ocorrer com maior freqüência durante o ano agrícola (SILVEIRA et al., 1985).

De acordo com BENEZ (2002), para aperfeiçoar o uso da máquina, o agricultor deve, inicialmente, realizar a análise operacional para verificar quais são as operações que representam as reais condições da propriedade.

O desempenho econômico do sistema tratorizado é diretamente proporcional a sua capacidade operacional, ou seja, quanto maior seu valor, menores serão os custos, melhorando economicamente o trabalho do sistema (MIALHE, 1974).

Pressupõe-se que os manejos de diferentes culturas de coberturas influenciam na taxa de decomposição da massa seca sobre a superfície do solo, refletindo na velocidade de deslocamento e, conseqüentemente, na capacidade de campo efetiva do conjunto utilizados nesses manejos.

O presente trabalho teve por objetivo estudar a produção e a taxa de decomposição de duas coberturas vegetais (crotalária juncea e mucuna-cinza) em três sistemas de manejo (rolo-faca, triturador de palhas e herbicida), e verificar qual equipamento proporciona melhor capacidade de campo efetiva.

\section{MATERIAL E MÉTODOS}

O experimento foi conduzido na área experimental do Laboratório de Máquinas e Mecanização Agrícola (LAMMA) da UNESP - Jaboticabal - SP, localizada nas coordenadas geodésicas: latitude $21^{\circ} 14^{\prime} \mathrm{S}$ e longitude $48^{\circ} 16^{\prime} \mathrm{W}$, com altitude média de $560 \mathrm{~m}$, declividade média de $4 \%$ e clima Cwa (subtropical), de acordo com a classificação de Köeppen. O solo da área experimental foi classificado como Latossolo Vermelho Eutroférrico, conforme o Sistema Brasileiro de Classificação de Solos (EMBRAPA, 1999).

O delineamento experimental foi de blocos casualizados, em esquema fatorial $3 \times 2$, originando seis tratamentos, com quatro repetições, totalizando 24 observações. Os tratamentos 
foram três métodos de manejo (rolo-faca, triturador de palhas e herbicida), em duas coberturas vegetais - crotalária juncea (Crotalária juncea L.) e mucuna-cinza (Stilozobium niveum L).

Cada parcela experimental ocupou área de $300 \mathrm{~m}^{2}(25 \times 12 \mathrm{~m})$, distribuídas em quatro blocos, e entre as parcelas, no sentido longitudinal, foi reservado espaço de $15 \mathrm{~m}$ destinado à realização de manobras, tráfego de máquinas e estabilização dos conjuntos.

$\mathrm{Na}$ semeadura, foram utilizadas as espécies e suas respectivas quantidades de sementes por unidade de área, conforme Tabela 1 .

TABELA 1. Descrição das culturas de cobertura utilizadas.

\begin{tabular}{lcccc}
\hline \multirow{2}{*}{ Espécie } & $\begin{array}{c}\text { Quantidade } \\
\left(\mathrm{kg} \mathrm{ha}^{-1}\right)\end{array}$ & Pureza & Germinação & Valor Cultural \\
\cline { 3 - 5 } & 25 & 98,5 & 76 & \\
\hline Crotalária (Crotalaria juncea L.) & 60 & 98,3 & 81 & 74,6 \\
Mucuna (Stilozobium niveum L. $)$ & & &
\end{tabular}

Utilizou-se fertilizante da fórmula 4-20-20 na dose de $150 \mathrm{~kg} \mathrm{ha}^{-1}$ na semeadura de crotalária e mucuna, no dia 6-4-2004, e herbicida gliphosate em pré-emergência, na dose de 3 e $6 \mathrm{~L} \mathrm{ha}^{-1}$ para o manejo químico das culturas de cobertura. Os manejos foram realizados após a ultima coleta da massa seca (125 dias após a semeadura).

Durante a instalação e a condução deste trabalho, foram utilizadas as máquinas e implementos descritos na Tabela 2.

TABELA 2. Equipamentos utilizados.

\begin{tabular}{|c|c|c|c|}
\hline Tratores & Marca/ Modelo & Potência & Características \\
\hline \multirow{3}{*}{ Tratores } & Ford 6600 & $63 \mathrm{~kW}(86 \mathrm{cv})$ & $4 \times 2$ \\
\hline & MF 620 & $77 \mathrm{~kW}(105 \mathrm{cv})$ & $4 \times 2$ TDA \\
\hline & MF 660 & $110 \mathrm{~kW}(150 \mathrm{cv})$ & $4 \times 2$ TDA \\
\hline Equipamentos & Marca/ Modelo & \multicolumn{2}{|c|}{ Características } \\
\hline Semeadora & Jumil 2650 Exacta & \multicolumn{2}{|c|}{ Quatro linhas de plantio, espaçadas de $0,5 \mathrm{~m}$. } \\
\hline Pulverizador & Jacto Condor & \multicolumn{2}{|c|}{ Tanque de $600 \mathrm{~L}, 24$ bicos tipo leque, largura de $12 \mathrm{~m}$. } \\
\hline Triturador & Jumil Trimax 2500 & \multicolumn{2}{|c|}{ Montado, largura de corte de $2,3 \mathrm{~m}}$. \\
\hline Rolo-faca & LAMMA & \multicolumn{2}{|c|}{$\begin{array}{l}\text { Montado, largura de corte de } 1,6 \mathrm{~m} \text {, cilindro de } 1 \mathrm{~m} \text { de } \\
\text { diâmetro, massa de } 1.360 \mathrm{~kg} \text { e distância entre facas de } 0,2 \mathrm{~m} \text {. }\end{array}$} \\
\hline
\end{tabular}

Para a determinação da massa seca, utilizou-se de um quadrado de ferro de $0,25 \mathrm{~m}^{2}$, tesoura de poda, estufa elétrica e balança de precisão de 0,01 g. As avaliações aos 30; 60; 97 e 125 dias após a semeadura (DAS) e aos 30; 51 e 71 dias após o manejo (DAM) foram feitas coletando-se uma amostra por parcela, secada em estufa elétrica a $70^{\circ} \mathrm{C}$ até atingir massa constante.

A largura efetiva de trabalho dos equipamentos foi medida diretamente sobre as passadas dos equipamentos nas parcelas e suas médias foram: rolo-faca $(1,60 \mathrm{~m})$, triturador de palhas $(2,15 \mathrm{~m}) \mathrm{e}$ herbicida $(12,00 \mathrm{~m})$.

A partir do espaço percorrido e do tempo de percurso medido por meio de cronômetro digital em todas as parcelas experimentais, determinou-se a velocidade real de deslocamento. No intuito de estabilizar as determinações, o movimento começava antes da baliza de início da parcela, e o tempo era cronometrado quando o referencial no trator coincidia sobre esta, e interrompido depois de decorridos os $25 \mathrm{~m}$ da parcela, ou seja, quando o referencial no trator coincidia com a baliza final.

Para cada conjunto, ao longo do trabalho, foram selecionadas marchas distintas, de acordo com a recomendação do fabricante (Tabela 3), no intuito de obter o melhor desempenho dos órgãos ativos dos equipamentos. 
TABELA 3. Relação de tratores e velocidades de trabalho.

\begin{tabular}{lcc}
\hline Trator & Manejo & Velocidade $\left(\mathrm{km} \mathrm{h}^{-1}\right)$ \\
\hline FORD 6600 (4x2) & Herbicida & 7,0 \\
MF 660 (4x2 TDA) & Rolo-faca & 7,5 \\
MF 620 (4x2 TDA) & Triturador de palhas & 7,0 \\
\hline
\end{tabular}

A capacidade de campo efetiva foi determinada segundo MIALHE (1974), com base na largura de trabalho real do equipamento e velocidade real de deslocamento proporcionada pela marcha selecionada em cada manejo.

Para o cálculo da capacidade de campo efetiva, utilizou-se da seguinte equação:

$$
\mathrm{Cce}=\frac{\mathrm{Lrt} \mathrm{Vrt}}{10}
$$

em que,

Cce - capacidade de campo efetiva, ha $\mathrm{h}^{-1}$;

Vrt - velocidade real de trabalho, $\mathrm{km} \mathrm{h}^{-1}$;

Lrt - largura real de trabalho do equipamento, $\mathrm{m}$, e

10 - fator de conversão.

\section{RESULTADOS E DISCUSSÃO}

Pela Tabela 4, observa-se que as parcelas nas quais seriam montados os manejos e as culturas de coberturas não afetaram a produção de massa seca da parte aérea aos 30 e 70 DAS (dias após semeadura). Para a massa seca coletada aos 97 DAS, houve diferença significativa entre as culturas de cobertura, com a crotalária apresentando maior quantidade de massa em relação à mucuna, fato esse relacionado à escassez de chuvas no período e ao rápido crescimento inicial da crotalária. Para as parcelas que receberiam os manejos, nota-se diferença significativa para o triturador de palhas em relação aos demais, sendo esse o de menor acúmulo de massa seca. Aos 125 DAS, houve semelhança estatística entre as parcelas nas quais seriam montados os manejos com triturador de palhas e rolo-faca, diferenciando-se da parcela que receberia o manejo com herbicida, que proporcionou maior quantidade de massa seca.

TABELA 4. Síntese de análise de variância e do teste de médias para massa seca da parte aérea das coberturas vegetais aos 30; 70; 97 e 125 DAS (dias após a semeadura).

\begin{tabular}{lcccc}
\hline \multirow{2}{*}{ Fatores } & \multicolumn{4}{c}{ Massa seca $\left(\mathrm{kg} \mathrm{ha}^{-1}\right)$} \\
\cline { 2 - 5 } & $30 \mathrm{DAS}$ & $70 \mathrm{DAS}$ & $97 \mathrm{DAS}$ & $125 \mathrm{DAS}$ \\
\hline Manejos & & & & \\
Triturador & $425 \mathrm{a}$ & $3.265 \mathrm{a}$ & $2.854 \mathrm{~b}$ & $3.222 \mathrm{~b}$ \\
Rolo-faca & $487 \mathrm{a}$ & $3.280 \mathrm{a}$ & $3.815 \mathrm{a}$ & $3.182 \mathrm{~b}$ \\
Herbicida & $482 \mathrm{a}$ & $3.655 \mathrm{a}$ & $3.902 \mathrm{a}$ & $3.512 \mathrm{a}$ \\
\hline Culturas & & & & \\
Mucuna & $459 \mathrm{a}$ & $3.318 \mathrm{a}$ & $3.107 \mathrm{~b}$ & $3.283 \mathrm{a}$ \\
Crotalária & $471 \mathrm{a}$ & $3.481 \mathrm{a}$ & $3.940 \mathrm{a}$ & $3.328 \mathrm{a}$ \\
\hline Teste F & & & & \\
Manejos (M) & $0,52^{\mathrm{NS}}$ & $1,27^{\mathrm{NS}}$ & $6,84^{* *}$ & $7,63^{* *}$ \\
Culturas (C) & $0,05^{\mathrm{NS}}$ & $0,52^{\mathrm{NS}}$ & $10,52^{* *}$ & $0,36^{\mathrm{NS}}$ \\
M x C & $0,05^{\mathrm{NS}}$ & $1,46^{\mathrm{NS}}$ & $2,14^{\mathrm{NS}}$ & $2,92^{\mathrm{NS}}$ \\
\hline C.V. $(\%)$ & 28,9 & 16,3 & 17,8 & 5,5 \\
\hline
\end{tabular}

Médias seguidas de mesmas letras minúsculas nas colunas não diferem entre si, pelo teste de Tukey, a $5 \%$ de probabilidade; ${ }^{* *}$ significativo $(\mathrm{p} \leq 0,01)$; C.V. - coeficiente de variação; ${ }^{\mathrm{NS}}$ - não-significativo. 
BARROS (2002), ao analisar a produção de matéria seca da parte aérea da crotalária e da mucuna-cinza aos 30; 60 e 90 dias após a semeadura, verificou que, nas duas primeiras avaliações, a crotalária foi superior estatisticamente à mucuna. As produções de matéria seca foram de $981 \mathrm{e}$ $415 \mathrm{~kg} \mathrm{ha}^{-1}$ aos 30 dias, e 8.175 e $5.092 \mathrm{~kg} \mathrm{ha}^{-1}$ aos 60 dias para crotalária e mucuna, respectivamente. Aos 90 dias após a semeadura, as culturas de coberturas não diferiram entre si.

Resultado similar foi encontrado por MACIEL (2002) para as culturas de coberturas crotalária e mucuna-cinza, com a primeira apresentando maior produção aos 30 e 60 dias após a semeadura. As produções de massa seca de crotalária foram de 6.065 e $5.628 \mathrm{~kg} \mathrm{ha}^{-1}$ aos 30 e 60 DAS e da mucuna de 2.537 e $2.921 \mathrm{~kg} \mathrm{ha}^{-1}$ aos 30 e 60 DAS, respectivamente. Porém, aos 90 DAS, a crotalária e a mucuna-cinza não diferiram quanto à produção da massa seca. $\mathrm{O}$ autor afirma que isso se deve ao rápido crescimento inicial da crotalária, mas que, ao final do ciclo, a mucuna consegue igualar a produção de massa seca.

De acordo com a Tabela 5, observa-se semelhança estatística entre os fatores estudados aos 30; 51 e 71 DAM (dias após manejo). A massa seca das culturas de cobertura não teve influência dos tratamentos até mais de dois meses após os manejos, apesar de as parcelas de triturador e rolofaca terem apresentado maior produção de massa ao final do ciclo. Esses dados possibilitam a utilização de qualquer equipamento de manejo apresentado neste trabalho, ou seja, pode-se utilizar o pulverizador (herbicida) que confere alta capacidade de campo efetiva (Tabela 6) ou, então, os equipamentos de manejo mecânico que apresentam menor capacidade de campo efetiva, porém independem das condições de umidade que o herbicida necessita para ser aplicado e translocado na planta.

Para BARROS (2002), as culturas de cobertura crotalária e mucuna-cinza diferiram após os manejos, apresentando 10.000 e $7.300 \mathrm{~kg} \mathrm{ha}^{-1}$ de massa seca no solo, respectivamente.

MACIEL (2002), avaliando diferentes sistemas de manejo (triturador de palhas, ceifadora de tambor e pulverizador), não encontrou diferença na quantidade de massa seca aos 30;60; 90 e 120 dias após a semeadura.

Observa-se que ocorreu aumento de massa seca após 30 DAM em relação aos 125 DAS, devido à presença de massa seca no solo antes do manejo, fazendo com que se acarretasse aumento aproximado de $700 \mathrm{~kg}$.

TABELA 5. Síntese de análise de variância e do teste de médias para massa seca da parte aérea das coberturas vegetais aos 30; 51 e 71 DAM (dias após manejo).

\begin{tabular}{|c|c|c|c|}
\hline \multirow{2}{*}{ Fatores } & \multicolumn{3}{|c|}{ Massa Seca $\left(\mathrm{kg} \mathrm{ha}^{-1}\right)$} \\
\hline & 30 DAM & $51 \mathrm{DAM}$ & $71 \mathrm{DAM}$ \\
\hline \multicolumn{4}{|l|}{ Manejos } \\
\hline Triturador & $4.145 \mathrm{a}$ & $3.052 \mathrm{a}$ & $2.371 \mathrm{a}$ \\
\hline Rolo-faca & $4.002 \mathrm{a}$ & $3.697 \mathrm{a}$ & $2.707 \mathrm{a}$ \\
\hline Herbicida & $4.015 \mathrm{a}$ & $3.987 \mathrm{a}$ & $2.542 \mathrm{a}$ \\
\hline \multicolumn{4}{|l|}{ Culturas } \\
\hline Mucuna & $4.048 \mathrm{a}$ & $3.663 \mathrm{a}$ & $2.629 \mathrm{a}$ \\
\hline Crotalária & $4.046 \mathrm{a}$ & $3.495 \mathrm{a}$ & $2.451 \mathrm{a}$ \\
\hline \multicolumn{4}{|l|}{ Teste F } \\
\hline Manejos (M) & $0,43^{\mathrm{NS}}$ & $3,08^{\mathrm{NS}}$ & $1,11^{\mathrm{NS}}$ \\
\hline Culturas (C) & $0,0002^{\mathrm{NS}}$ & $0,29^{\mathrm{NS}}$ & $0,94^{\mathrm{NS}}$ \\
\hline $\mathrm{M} \times \mathrm{C}$ & $2,37^{\mathrm{NS}}$ & $2,41^{\mathrm{NS}}$ & $0,50^{\mathrm{NS}}$ \\
\hline C.V. (\%) & 7,2 & 21,5 & 17,7 \\
\hline
\end{tabular}

Médias seguidas de mesmas letras minúsculas nas colunas não diferem entre si, pelo teste de Tukey, a $5 \%$ de probabilidade; C.V. - coeficiente de variação; ${ }^{\mathrm{NS}}$ - não-significativo. 
Na Figura 1, é apresentada a taxa de decomposição da massa seca após o manejo e observase que, logo após o manejo até os 51 dias, a decomposição é de aproximadamente $18 \mathrm{~kg} \mathrm{ha}^{-1} \mathrm{dia}^{-1}$, enquanto, a partir de 51 dias, a decomposição se acelera, ocorrendo taxa acima de $50 \mathrm{~kg} \mathrm{ha}^{-1}$. BARROS (2002) observou que a decomposição foi aos 55 dias após o manejo para a crotalária e mucuna-cinza foi semelhante, ou seja, $43,3 \%$ e $39,1 \%$ da massa seca original, respectivamente.

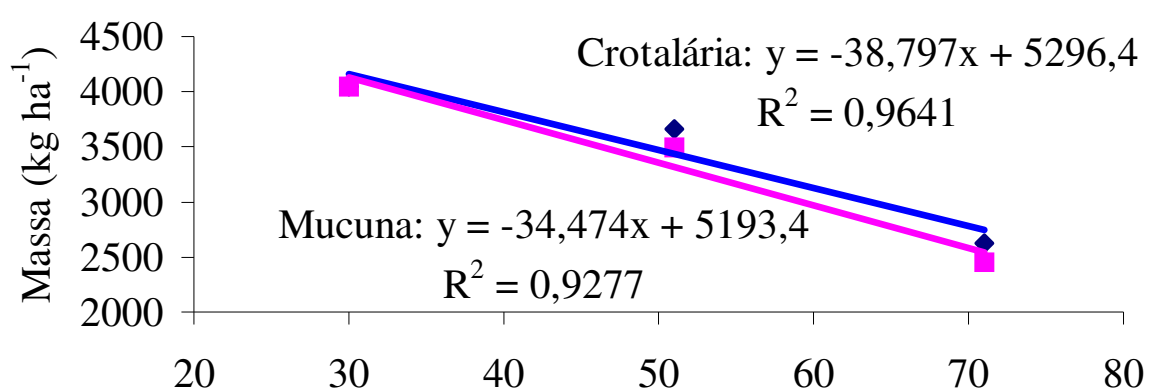

Dias após manejo

- Mucuna $\square$ Crotalária

FIGURA 1. Taxa de decomposição da massa seca após manejo.

Pela Tabela 6, nota-se semelhança entre as culturas de cobertura para as variáveis velocidade real de deslocamento e capacidade de campo efetiva. Para o fator manejo, observa-se diferença estatística entre as velocidades, mostrando que o manejo com rolo-faca permitiu desenvolver maior velocidade, seguido do herbicida e triturador de palhas, respectivamente. A variável capacidade de campo efetiva diferiu estatisticamente, sendo o manejo com herbicida o de maior valor, devido sua maior largura real de trabalho, seguido pelo triturador de palhas e rolo-faca.

MACIEL (2002) encontrou maior velocidade de deslocamento para o triturador de palhas e o pulverizador, discordando deste experimento. Entretanto, o autor obteve maior capacidade de campo efetiva para o pulverizador (herbicida), devido a sua maior largura de trabalho, o que também ficou evidenciado neste experimento.

TABELA 6. Síntese de análise de variância e do teste de médias para velocidade real de deslocamento $\left(\mathrm{km} \mathrm{h}^{-1}\right)$ e capacidade de campo efetiva $\left(\mathrm{hah}^{-1}\right)$.

\begin{tabular}{lcc}
\hline Fatores & Velocidade & Cce \\
\hline Manejo & & $1,4 \mathrm{~b}$ \\
Triturador & $6,6 \mathrm{c}$ & $1,1 \mathrm{c}$ \\
Rolo-faca & $7,1 \mathrm{a}$ & $8,4 \mathrm{a}$ \\
Herbicida & $6,8 \mathrm{~b}$ & $3,6 \mathrm{a}$ \\
\hline Cultura & & $3,6 \mathrm{a}$ \\
Mucuna & $6,8 \mathrm{a}$ & $35.954,33^{* *}$ \\
Crotalária & $6,8 \mathrm{a}$ & $1,00^{\mathrm{NS}}$ \\
Teste F & $65,28^{* *}$ & $2,33^{\mathrm{NS}}$ \\
Manejo (M) & $0,79^{\mathrm{NS}}$ & 1,69 \\
Cultura (C) & $2,42^{\mathrm{NS}}$ & 1,34 \\
M x C & 1,34
\end{tabular}

Médias seguidas de mesmas letras minúsculas nas colunas não diferem entre si, pelo teste de Tukey, a $5 \%$ de probabilidade; ${ }^{* *}$ significativo ( $\left.\leq \leq 0,01\right) ;$ C.V. coeficiente de variação; ${ }^{\text {NS }}$ não-significativo. 


\section{CONCLUSÕES}

A produção de massa seca foi igual nas duas culturas, e as taxas de decomposição da crotalária e da mucuna foram semelhantes para os três manejos estudados.

O manejo das culturas de cobertura com pulverizador (químico) proporcionou maior capacidade de campo efetiva.

\section{REFERÊNCIAS}

BARROS, F.L. Semeadura direta da cultura do milho (Zea mays L.) em função de diferentes manejos da cobertura vegetal. 2002. 63 f. Monografia (Graduação em Agronomia) - Faculdade de Ciências Agrárias e Veterinárias, Universidade Estadual Paulista, Jaboticabal, 2002.

BENEZ, S.H. Racionalização do uso de máquinas em sistema de plantio direto. In: ENCONTRO NACIONAL DE PLANTIO DIRETO NA PALHA, 1., 2002, Águas de Lindóia. Anais... Águas de Lindóia: Nobel, 2002. p.8.

EMBRAPA. Serviço Nacional de Produção de Informações. Sistema plantio direto. Brasília, 1998. $248 \mathrm{p}$.

EMBRAPA. Centro Nacional de Pesquisas de Solos. Sistema brasileiro de classificação de solos. Brasília, 1999. 412 p.

MACIEL, R.F. Manejo das culturas de crotalária (Crotalária juncea L.) e mucuna-cinza (Stizolobuim niveum) visando ao plantio direto. 2002. $53 \mathrm{f}$. Monografia (Graduação em Agronomia) - Faculdade de Ciências Agrárias e Veterinárias, Universidade Estadual Paulista, Jaboticabal, 2002.

MIALHE, L. G. Manual de mecanização agrícola. São Paulo: Agronômica Ceres, 1974. 301 p. MOODY, J.E.; SHEAR, G.M.; JONES JUNIOR, J.N. Growing corn without tillage. Soil Science Society of American Procedure, Madison, v.6, n.7, p.516, 1961.

SILVA, J.A.A.; DONADIO, L.C.; CARLOS, J.A.D. Adubação verde em cítrus. Jaboticabal: Funep, 1999. 37 p.

SILVEIRA, G.M.; KURACHI, S.A.; FUJIWARA, M. Métodos mecânicos e químicos de controle de ervas daninhas em cafezal. Bragantia, Campinas, v.44, n.1, p.173-8, 1985. 